\title{
Trayectorias de victimización escolar: características y factores de riesgo en adolescentes chilenos*
}

\author{
Trajectories of school victimization: Characteristics \\ and risk factors in chilean teenagers
}

\author{
Christian Berger Silva ** \\ Universidad Alberto Hurtado, Santiago, Chile
}

SICI: 2011-2777(201203)11:1<103:TVECFR>2.0.CO;2-Q

Para citar este artículo: Berger, C. (2012). Trayectorias de victimización escolar: características y factores de riesgo en adolescentes chilenos. Universitas Psychologica, 11(1), 103-118.

* Patrocinado por la Fundación Andes.

** Profesor Auxiliar. Facultad de Psicología. Universidad Alberto Hurtado, Chile. E-mail: cberger@ uahurtado.cl

\section{RES UMEN}

La victimización es un tema de creciente preocupación escolar. No obstante, no hay claridad respecto del perfil de los estudiantes victimizados, ni de los potenciales factores de riesgo. El presente estudio adopta un enfoque ecológico y un modelo longitudinal, siguiendo a más de 600 estudiantes chilenos de $5^{\text {to }}$ y $6^{\text {to }}$ año, por un período de un año. Se identifican distintas trayectorias asociadas a la victimización (estables, nuevas, resilientes y no víctimas), definiendo perfiles psicosociales asociados a estas. Asimismo, identifica factores de riesgo para hombres y mujeres desde las perspectivas de pares y profesores. Se discuten implicancias conceptuales y metodológicas, así como posibles líneas de prevención e intervención

\section{Palabras clave autor:}

Victimización escolar, trayectorias, factores de riesgo.

Palabras clave descriptores:

Enfoque ecológico, modelo longitudinal, estudiantes, investigación cuantitativa, psicología educativa.

\footnotetext{
A B S T R AC T

Victimization is a growing concern in the school context. Nevertheless, clarity about the profile of victimized students and potential risk factors is still lacking. This longitudinal study followed over 600 Chilean students for a year within an ecological perspective, and identified several trajectories linked to victimization (stable, new, resilient and nonvictims), defining psychosocial profiles. Risk factors for men and women are identified, by taking into account the opinions of peers and teachers. Conceptual and methodological issues are discussed, along with possible prevention and intervention lines.

Key words author:

School victimization, trajectories, risk factors.

Key words plus:

Ecological approach, longitudinal model, students, quantitative research, educational psychology.
} 
La violencia en las escuelas se ha transformado en una preocupación central para profesionales e investigadores (Elias \& Zins, 2003; Miller \& Krauss, 2008; Pellegrini, 1998). Más específicamente, las agresiones entre pares (también llamada matonaje, hostigamiento, acoso escolar o bullying), una manifestación específica de violencia en las escuelas, ha sido foco de creciente atención. La literatura muestra ya un importante cuerpo de evidencia acumulada respecto de los efectos negativos asociados a este tipo de relaciones interpersonales, tanto para los agresores como para las víctimas (Berger \& Lisboa, 2008; Graham, Bellmore \& Juvonen, 2003). Entre los efectos para los y las estudiantes víctimas de matonaje, se han descrito síntomas depresivos, ansiedad y aislamiento (Hodges \& Perry, 1999; Kumpulainen et al., 1998; Olweus, 1993), pasividad y sumisión (Olweus, 1993), dificultades en el ámbito académico (Buhs, Ladd \& Herald, 2006; Nansel, Haynie \& Simons-Morton, 2003; Tam, 2008), así como conductas antisociales (Paul \& Cillessen, 2003; Swearer \& Tam, 2003) y rechazo de parte de sus pares (Olweus, 1993).

La victimización -o su contracara, el bullying,es una relación de abuso en la cual uno o más individuos intimidan, agreden y abusan de otro, existiendo un desequilibro de poder entre ambos (Graham \& Juvonen, 1998; Olweus, 1993). La experiencia de ser victimizado supone la incapacidad de dejar la posición de víctima. Más aún, perspectivas ecológicas de la violencia entre pares ponen énfasis en el rol que el grupo y los observadores tienen frente a la victimización, dándole sentido y validándola (Berger, 2008; Berger \& Rodkin, 2009; García \& Madriaza, 2005; Lisboa \& Koller, 2008).

Diversos estudios señalan que entre el $10 \%$ y $20 \%$ de la población escolar a nivel mundial sería víctima de violencia por parte de sus pares (Ortega \& Mora-Merchán, 1997; Pellegrini, 1998; Rodkin \& Berger, 2008; Schwartz, Proctor \& Chien, 2001). Las tasas más altas de victimización se observan entre el $6^{\text {to }}$ y $7^{\text {mo }}$ grado, aproximadamente 12 y 13 años (Graham \& Juvonen, 1998; Orpinas \& Horne, 2006). En Chile, la Segunda Encuesta Nacional de Violencia en el Ámbito Escolar (Ministerio del Interior, 2008) señala que un $26.3 \%$ de los estudiantes declaró haber sido víctima de algún tipo de agresión -no necesariamente bullying- principalmente de carácter psicológico y/o físico.

Existe evidencia respecto de las diferencias existentes entre estudiantes víctimas de matonaje (Boulton, 1999; Goldbaum, Craig, Pepler \& Connolly, 2003; Holt \& Espelage, 2003; Paul \& Cillessen, 2003). Si bien los agresores son mayormente hombres, las víctimas se distribuyen de manera equitativa entre hombres y mujeres (Espelage, Mebane \& Swearer, 2004; Rodkin \& Berger, 2008; Scheithauer, Hayer, Petermann \& Jugert, 2006; Schwartz et al., 2001; Solberg \& Olweus, 2003; Veenstra et al., 2007). Rodkin y Berger (2008) plantean que las implicancias de ser victimizado pueden ser distintas para hombres y para mujeres. En su estudio con estudiantes de $4^{\text {to }}$ y $5^{\text {to }}$ grado encontraron que, si bien los hombres que eran victimizados por sus pares respondían a la imagen clásica de un niño tímido, débil y rechazado, mujeres que eran victimizadas por hombres presentaban un perfil distinto caracterizado por niveles de estatus social normal o alto. Otros estudios han encontrado diferencias entre estudiantes víctimas de matonaje en relación con sus estrategias de resolución de conflictos (Owens, Daly \& Slee, 2005), sus actitudes y respuestas ante comportamientos agresivos (Tapper \& Boulton, 2005), y sus atribuciones respecto de su situación (Troop-Gordon \& Ladd, 2005). Sin embargo, existe consenso entre los investigadores en que definir un perfil claro de víctima es de gran complejidad, pues la victimización no responde solo a variables individuales sino a cómo éstas interactúan con el contexto social específico (Berger \& Lisboa, 2008).

Identificar a los estudiantes víctimas de matonaje es una tarea difícil. En muchos casos la victimización se da en contextos ocultos y, por tanto, se reproduce el círculo del silencio propio a las experiencias de abuso (Barudy, 1999). Unnever y Cornell (2004) reportaron que, entre estudiantes de $5^{\text {to }}$ a $8^{\text {vo }}$ año, el $25 \%$ de las víctimas de matonaje no informó a nadie de su situación y el 40 \% no informó a ningún adulto. Lo anterior está relacionado también con otro aspecto que ha sido poco investigado: la estabilidad de la victimización. Rodkin y Berger (2008) encontraron que más del $50 \%$ de los estu- 
diantes categorizados por sus pares como víctimas, mantuvieron esta categorización después de seis meses. Camodeca, Goossens, Terwogt y Schuengel (2002) reportaron que niños victimizados mantuvieron esta situación en mayor proporción que niñas. Kochenderfer-Ladd (2003) encontró que estudiantes que eran rechazados por sus pares tenían una mayor probabilidad de mantener su situación de victimización. Los resultados de estos estudios muestran que la victimización no es una situación aislada y momentánea, sino que puede mantenerse en el tiempo. Por otra parte, el importante porcentaje de niños y niñas que sale de dicha situación abre un amplio campo de investigación e intervenciones, al identificar aquellos factores que permiten a un estudiante salir de la posición de víctima. No obstante lo anterior, generalmente los estudios han identificado a las víctimas de manera transversal, asumiendo a la victimización como una posición social estática. Desde esta perspectiva, se asume como equivalente a un estudiante que ha sido victimizado a lo largo del tiempo de otro que solo recientemente se ha transformado en víctima. Asimismo, se asume como igual a quien nunca ha sido victimizado que quien ha dejado dicha posición social. En el presente estudio se incluye la noción de trayectorias de victimización (Goldbaum et al., 2003) con el objetivo de incluir el carácter dinámico de esta e integrar la diversidad en dicha experiencia en relación con su estabilidad o cambio en el tiempo y potenciales factores protectores y de riesgo en relación a las trayectorias.

La investigación respecto de los factores de riesgo y protectores ha identificado algunas características individuales asociadas a una posterior victimización. En 1993 Olweus retrató a estos niños como ansiosos, inseguros, sensibles, callados y cautelosos, lo cual ha sido confirmado por otros estudios (Egan \& Perry, 1998; Hodges \& Perry, 1999). Paul y Cillessen (2003) encontraron que, en $4^{\text {to }}$ y $5^{\text {to }}$ grado, niños solitarios, ansiosos y que presentaban conductas disruptivas eran más proclives a ser victimizados durante su adolescencia temprana. Kochenderfer-Ladd (2003) encontró que niveles elevados de agresividad en edades tempranas también constituye un factor de riesgo. De hecho, diversos estudios han identificado un subgrupo de víctimas con elevados índices de agresividad (Pellegrini, Bartini \& Brooks, 1999; Swearer \& Tam, 2003). En términos más generales, diversos estudios han mostrado que niños y niñas que no responden a lo normativo, que escapan del promedio en un contexto determinado, están en mayor riesgo de ser victimizados (Bierman, 2004; Sullivan, Clearly \& Sullivan, 2005). En la misma línea, se han observado los espacios de intimidad y la integración social como factores de riesgo o protectores a nivel interpersonal (Berger \& Rodkin, 2009; Hartup, 1996; Hodges, Malone \& Perry, 1997; Hodges \& Perry, 1999; Rodkin \& Hodges, 2003).

Considerando la evidencia acumulada hasta la fecha e intentando ampliar el conocimiento sobre las dinámicas de victimización e iluminar posibles estrategias preventivas y promocionales de bienestar, el presente estudio se propone responder a cuatro preguntas centrales: (a) ¿Quiénes son los y las estudiantes victimizados por sus pares? (b) ¿Existen características o perfiles socioconductuales asociados a distintas trayectorias de victimización? (c) ¿Es la victimización una característica estable en el tiempo? Y, por último, (d) ¿Qué características constituyen factores de riesgo para la victimización?

\section{Método}

Con el fin de responder a dichos objetivos, el presente estudio adoptó un diseño longitudinal (dos mediciones con un periodo intermedio de un año) e integró información de distintas fuentes de reporte (pares, profesores y autorreporte) sobre la victimización y características socioconductuales de los participantes.

\section{Muestra}

La muestra total del estudio fue de 647 estudiantes (324 mujeres) de $5^{\text {to }}$ y $6^{\text {to }}$ año de enseñanza general básica de cuatro escuelas de Santiago (Chile). Se obtuvo consentimiento parental activo para todos los participantes. La primera medición incluyó a 576 estudiantes, de los cuales el $92.5 \%$ fue autorizado por sus padres y otros 41 participantes no estuvie- 
ron presentes en la medición; se recolectaron datos para 492. Análisis comparativos mostraron que los estudiantes ausentes eran menos populares y con menores índices de conductas prosociales según sus pares $(t s(532)=2.93$ y 3.25 respectivamente, $p<0.01)$. En la segunda medición $(N=591)$, $97.5 \%$ (576) fueron autorizados por sus padres y 32 estuvieron ausentes. Se recolectaron datos para 544 estudiantes y los análisis comparativos no mostraron diferencias significativas entre estudiantes presentes y ausentes. Integrando ambas mediciones se obtuvo información para 479 estudiantes ( $48.6 \%$ mujeres). Los estudiantes que dejaron el estudio en la segunda medición presentaron mayores índices de agresividad y mayor rechazo de parte de sus pares $(t s=2.08$ y 2.45 respectivamente, $p<0.05)$.

\section{Recolección de la información}

Los instrumentos se aplicaron en dos mediciones con un año de intervalo, de manera colectiva, durante el horario normal de clases (45 min).

Se aseguró la confidencialidad de las respuestas y el carácter voluntario de la participación en el estudio mediante consentimiento parental activo y asentimiento de los participantes. Los estudiantes respondieron cuestionarios sobre sus compañeros respecto de una serie de características sociales y conductuales. Profesores evaluaron a sus estudiantes y estos reportaron sobre sí mismos respecto de varias características socioconductuales. Durante la aplicación, un investigador leía en voz alta los cuestionarios mientras otros dos monitoreaban el trabajo de los participantes, respondiendo preguntas. El profesor se mantuvo en la sala respondiendo el cuestionario en su versión para profesores. Todos los aspectos éticos de la investigación fueron aprobados por el comité de ética de la universidad patrocinante y por los directivos de las distintas escuelas.

\section{Instrumentos}

\section{Victimización}

Las víctimas fueron identificadas utilizando el consenso de pares. Se calculó un índice de victimización para cada participante con base en el reporte de sus compañeros (número de nominaciones recibidas como víctima sobre el potencial máximo de nominaciones, esto es, compañeros presentes - 1). Se adoptó tanto una estrategia de análisis centrada en las personas como otra centrada en las variables (Cairns \& Rodkin, 1998). Respecto del análisis centrado en las personas, existe debate respecto de cómo identificar a las víctimas (Nylund, Bellmore, Nishina \& Graham, 2007), ya sea utilizando criterios de corte basado en percentiles, en desviaciones estándar o bien en grupos extremos, todos ellos con fortalezas y debilidades. Para el presente estudio, se categorizó a los participantes como víctimas o no víctimas (Graham, Bellmore \& Mize, 2006), considerando como criterio de corte una desviación estándar sobre el promedio en el puntaje de victimización en relación con el total de participantes, en cada medición. Si bien esta categorización polar puede parecer simplista, este criterio permite obtener una muestra representativa de estudiantes victimizados, considerando la distribución anormal de la variable victimización con una alta concentración de participantes con un valor de 0 (es decir, sin nominaciones como víctimas). No obstante, no es excluyente en relación con las dificultades reportadas respecto a la identificación de víctimas (Berger, Rodkin \& Karimpour, 2008; Graham \& Juvonen, 1998). Por otra parte, se realizaron análisis desde una estrategia centrada en la variable, considerándola continua. Desde esta perspectiva, se analizaron variables asociadas y predictoras de la victimización, utilizando modelos de regresión múltiple.

\section{Nominación de pares}

Los estudiantes respondieron un cuestionario en el cual podían nominar hasta seis compañeros o compañeras que mejor calzaran con una serie de descriptores sociales y conductuales. Para cada participante se calculó en cada ítem un coeficiente de nominación (procedimiento similar al índice de victimización antes presentado). Considerando que las nominaciones de pares no siguen una distribución normal (Boivin, Dodge \& Coie, 1995), los valores fueron transformados (log10; se eliminaron valores 
negativos sumando una constante) y luego estandarizados (Cillessen \& Mayeux, 2004; Rodkin \& Berger, 2008). Los ítems individuales fueron luego sometidos a un análisis factorial (MLE, rotación Varimax). Tres factores explicaron el $58.8 \%$ de la varianza: Agresividad (ítems "comienza peleas", "se ríe de los otros", "se mete en problemas" e "ignora a los otros"); Conductas Prosociales (ítems "es amable", "coopera”, "otros quieren ser como él/ella" y "otros escuchan lo que él/ella dice"); Prominencia Social (ítems "no popular (-)", "bacán”, "atlético" y "popular"). Los índices de consistencia interna $(\alpha)$ fueron $0.839,0.801$ y 0.759 , respectivamente.

\section{Estatus social}

Se distinguió entre preferencia social y popularidad como dos marcadores distintos de estatus social (Berger \& Rodkin, 2009; LaFontana \& Cillessen, 2002). Los participantes fueron consultados sobre aquellos compañeros y compañeras con las que más les gustaría jugar y con los que menos les gustaría jugar. La preferencia social fue calculada como el puntaje de cada participante en ser considerado con quien más se quiere jugar, menos el puntaje obtenido como con quien menos se quiere jugar (Jiang \& Cillessen, 2005; Rodkin \& Berger, 2008). De manera similar, el constructo de popularidad se calculó restando del puntaje obtenido como popular, según el reporte de los pares, el puntaje obtenido como no popular. Ambos marcadores de estatus mostraron altas correlaciones entre ambas mediciones $(r=0.74$ y 0.8$)$.

\section{Escala de competencias interpersonales - profesores (ICS-T)}

El ICS en su versión para profesores (T); es un cuestionario de 18 ítems (incluyendo dos distractores y algunos ítems con valores inversos) de tipo Likert (7 puntos) que el profesor contesta para cada uno de los estudiantes de su clase. El ICS-T entrega puntuaciones en las subescalas de Popularidad (ítems "popular con los niños", "popular con las niñas" y "tiene muchos amigos"), Olympian/Competencia física (ítems "bueno en los deportes", "atractivo físicamente" y "siempre gana”), Agresión (ítems "siempre discute", "se mete en problemas" y "siempre pelea"), Dificultades psicológicas (ítems "siempre está ansioso" y "llora mucho") y Competencia académica (ítems "bueno en matemáticas" y "bueno en lenguaje"). Además, el cuestionario incluye una subescala llamada Filiación (ítems "siempre sonríe" y "siempre es amistoso"), que en esta versión fue aumentada con tres ítems adicionales ("ayuda a sus compañeros", "tiene muchos amigos" y "coopera") y renombrada como Conductas prosociales. Los índices de consistencia interna $(\alpha)$ en ambas mediciones fueron: Popularidad (0.83 y 0.76), Olympian ( 0.71 y 0.57$)$, Agresividad (0.85 y 0.86), Dificultades psicológicas $(0.64$ y 0.52$)$, Conductas prosociales (0.84 y 0.86) y Competencias académicas $(0.57$ y 0.52). Los coeficientes de estabilidad de estas características estuvieron entre 0.29 y 0.55 (ps $<0.001)^{1}$.

\section{Escala de competencias interpersonales- autorreporte (ICS-S)}

La versión autorreporte del ICS es una escala tipo Likert (5 puntos) de 17 ítems (algunos con valores inversos) paralelos a los ítems de la versión para profesores. Las subescalas de esta versión son similares a las presentadas para la versión de profesores, con la excepción de la subescala Conductas prosociales que no fue aumentada (se mantuvo la original, Filiación). Asimismo, la subescala Dificultades psicológicas no está presente en esta versión. Los índices de consistencia interna $(\alpha)$ para ambas mediciones fueron: Popularidad (0.63 y 0.65), Olympian $(0.46$ y 0.54), Agresividad (0.53 y 0.67), Filiación (0.18 y 0.17 ) y Competencias académicas $(0.25$ y 0.3$)$, y sus coeficientes de estabilidad fluctuaron entre $0.36 \mathrm{y}$ $0.51(p<0.001)$. La versión en español utilizada en este estudio (ICS-T y ICS-S) fue utilizada previamente en estudios con población hispanoparlante en Estados Unidos (Rodkin \& Berger, 2008) y adaptada a través de una traducción al español y luego

1 Estudios han mostrado validez convergente del ICS-T con datos observacionales y validez predictiva en un periodo de ocho años respecto de ajuste adulto, deserción escolar y paternidad adolescente (Cairns, Leung, Gest \& Cairns, 1995). 
TABLA 1

Características de víctimas y no víctimas (promedios) según reporte de pares, profesores y autorreporte, en ambas mediciones

\begin{tabular}{|c|c|c|c|c|c|c|c|}
\hline \multirow[b]{2}{*}{ Reporte } & \multirow[b]{2}{*}{ Característica } & \multicolumn{3}{|c|}{ Tiempo 1} & \multicolumn{3}{|c|}{ Tiempo 2} \\
\hline & & $\begin{array}{l}\text { Víctimas } \\
\mathrm{N}=75\end{array}$ & $\begin{array}{l}\text { No víctimas } \\
N=501\end{array}$ & $t$ & $\begin{array}{l}\text { Víctimas } \\
N=104\end{array}$ & $\begin{array}{l}\text { No víctimas } \\
\mathrm{N}=487\end{array}$ & $t$ \\
\hline \multirow{5}{*}{ Pares } & Agresión & 0.68 & -0.1 & $-6.59 * *$ & 0.47 & -0.01 & $-4.41 * *$ \\
\hline & Prominencia social & -0.22 & 0.03 & $2.03 *$ & -0.17 & 0.18 & $3.4^{*}$ \\
\hline & Conducta prosocial & -0.13 & 0.02 & 1.19 & -0.01 & 0.11 & 1.06 \\
\hline & Preferencia social & -0.99 & 0.15 & $9.98 * *$ & -0.85 & 0.23 & $10.41 * *$ \\
\hline & Popularidad & -0.79 & 0.12 & $7.67 * *$ & -0.83 & 0.17 & $9.49 * *$ \\
\hline \multirow{6}{*}{ Profesores } & Agresión & 0.56 & -0.08 & $-5.2 * *$ & 0.16 & -0.03 & -1.64 \\
\hline & Popularidad & -0.38 & 0.06 & $3.43 * *$ & -0.54 & 0.13 & $5.94 * *$ \\
\hline & "Olympian" & -0.28 & 0.04 & $2.52 *$ & -0.49 & 0.12 & $5.31 * *$ \\
\hline & Conducta prosocial & -0.42 & 0.06 & $3.85 * *$ & -0.43 & 0.1 & $4.59 * *$ \\
\hline & $\begin{array}{l}\text { Competencias } \\
\text { académicas }\end{array}$ & -0.37 & 0.05 & $3.35 * *$ & -0.29 & 0.07 & $3.12 *$ \\
\hline & Dificultades psicológicas & 0.59 & -0.09 & $-5.5 * *$ & 0.52 & -0.13 & $-5.7 * *$ \\
\hline \multirow{5}{*}{ Autorreporte } & Agresión & 0.21 & -0.03 & -1.86 & 0.36 & -0.08 & $-3.88 * *$ \\
\hline & Popularidad & -0.44 & 0.07 & $3.85 * *$ & -0.55 & 0.12 & $6.04 * *$ \\
\hline & "Olympian" & -0.22 & 0.03 & $1.96^{*}$ & -0.34 & 0.07 & $3.71 * *$ \\
\hline & $\begin{array}{l}\text { Competencias } \\
\text { académicas }\end{array}$ & 0.02 & -0.00 & $<1$ & -0.18 & 0.04 & $1.96^{*}$ \\
\hline & Filiación & -0.33 & 0.05 & 2.94* & -0.39 & 0.08 & $4.21 * *$ \\
\hline
\end{tabular}

$* * p<0.01 ; * p<0.05$

Fuente: elaboración propia

traducida nuevamente al inglés (back-translation) para comprobar su concordancia.

\section{Resultados}

\section{Análisis centrado en los individuos}

A nivel descriptivo, $12 \%$ de los participantes durante la primera medición y $16 \%$ durante la segunda fueron categorizados como víctimas, con base en el reporte consensuado de pares. Comparaciones simples entre víctimas y no víctimas en una serie de características reportadas por pares, profesores y por los propios estudiantes, muestra un perfil desadaptativo de las víctimas, con bajos índices de estatus social, aceptación y conductas prosociales, y elevados índices de agresividad y dificultades psicosociales, como puede observarse en la Tabla 1, diferencias que se mantuvieron en la segunda medición. En ambas mediciones las víctimas se distribuyeron de manera equilibrada por género $\left(\chi^{2}<1\right.$ y $\left.1,42, n s\right)$ y grado $\left(\chi^{2}<1\right.$ y $\left.1,35, n s\right)$.

Los participantes fueron clasificados según sus trayectorias de victimización como estables (estudiantes identificados como víctimas en ambos momentos, $N=42,8.1 \%$ ), nuevas (estudiantes que pasaron de no ser victimizados a serlo en la segunda medición, $N=51,9.8 \%$ ), resilientes (estudiantes que pasaron de ser víctimas en la primera medición a no serlo en la segunda, $N=25,4.8 \%$ ) y no víctimas (estudiantes no victimizados en ambas mediciones, $N=402,77.3 \%$ ). Si bien no se observaron diferencias significativas en la distribución de estas categorías por género y grado $\left(\chi^{2}=2.31\right.$ y $\left.6.16, n s\right)$, las mayores diferencias se observaron en las víctimas estables que fueron mayormente hombres $(59.5 \%)$ y en las resilientes que fueron mayormente mujeres (56 \%). 
Los estudiantes fueron comparados con base en sus trayectorias de victimización sobre las variables reportadas por sus pares, profesores y autorreportadas. Se realizaron distintas MANOVA (Wilks' lambda) con variables agrupadas por característica, integrando las distintas fuentes de reporte. Para todas las características se encontraron diferencias significativas. Las características fueron Agresividad $(F=3.57, p<0.01)$, Estatus social (incluyendo popularidad prominencia y preferencia social; $F=4.88, p<0.001$ ), Conductas prosociales $(F=4.17, p<0.01)$, Dificultades psicosociales (dificultades psicológicas, filiación; $F=3.81, p<0.01)$ y Competencia académica $(F=4.94, p<0.001)$.

A continuación se realizaron análisis univariados como seguimiento a los resultados significativos de las pruebas multivariadas. La Tabla 2 muestra los perfiles en la segunda medición de las víctimas (ANOVA), según su clasificación. Como se puede observar, los participantes que pasaron de no ser victimizados a serlo al momento de la medición (víctimas nuevas) presentan un perfil disfuncional, con altos niveles de Agresividad según las distintas fuentes de reporte (pares, profesores y autorreporte) y menores índices de Estatus social (prominencia, popularidad, preferencia y filiación). Las víctimas estables (victimizados en ambas mediciones) presentan un perfil disfuncional similar a las víctimas nuevas, con niveles más bajos de Agresividad e indicadores más bajos de Estatus social. Por otra parte, si bien las víctimas resilientes (aquellos participantes que dejaron su estatus de víctimas en la segunda medición) presentan un perfil con puntajes elevados en Estatus social y Conductas prosociales, también presentan elevados índices de Agresión y son percibidas por sus profesores como presentando Dificultades psicológicas y con un bajo Desempeño académico. Como se esperaba, participantes no victimizados presentan indicadores promedio en todas las variables.

TABLA 2

Características de las distintas categorías de víctimas (promedios y desviaciones estándar) según reporte de pares, profesores y autorreporte, en segunda medición

\begin{tabular}{|c|c|c|c|c|c|c|}
\hline \multirow[t]{2}{*}{ Reporte } & \multirow[t]{2}{*}{ Característica } & \multicolumn{4}{|c|}{ Víctimas } & \multirow[b]{2}{*}{$\mathrm{F}$} \\
\hline & & no víctimas & resilientes & nuevas & estables & \\
\hline \multirow{5}{*}{ Pares } & Agresión & $-0.03(0.89)$ & $0.67(1.45)$ & $0.70(1.51)$ & $0.31(1.03)$ & $11.34 * *$ \\
\hline & Prominencia social & $0.18(0.93)$ & $0.57(1.33)$ & $0.14(1.12)$ & $-0.39(0.73)$ & $6.1 * *$ \\
\hline & Conducta prosocial & $0.13(1.05)$ & $0.25(1.02)$ & $0.04(0.8)$ & $-0.07(0.81)$ & $<1$ \\
\hline & Preferencia social & $0.25(0.95)$ & $0.21(0.95)$ & $-0.74(0.96)$ & $-0.88(1.07)$ & $31.05 * *$ \\
\hline & Popularidad & $0.17(0.89)$ & $0.55(1.2)$ & $-0.46(1.42)$ & $-1.16(0.97)$ & $30.27 * *$ \\
\hline \multirow{6}{*}{ Profesores } & Agresión & $-0.1(0.96)$ & $0.74(1.11)$ & $0.3(1.17)$ & $0.07(0.96)$ & $6.7 * *$ \\
\hline & Popularidad & $0.11(0.96)$ & $0.54(0.87)$ & $-0.37(1)$ & $-0.72(0.85)$ & $12.91 * *$ \\
\hline & "Olympian" & $0.09(0.93)$ & $0.46(1)$ & $-0.24(1.05)$ & $-0.73(1.09)$ & $10.65 * *$ \\
\hline & Conducta prosocial & $0.1(0.97)$ & $0.37(0.79)$ & $-0.51(0.97)$ & $-0.44(1.13)$ & $8.64 * *$ \\
\hline & Competencia académica & $0.1(0.96)$ & $-0.42(0.9)$ & $-0.42(1.08)$ & $-0.07(1.07)$ & $5.38 * *$ \\
\hline & Dificultades psicológicas & $-0.13(0.97)$ & $0.22(0.64)$ & $0.53(1.11)$ & $0.67(0.96)$ & $12.32 * *$ \\
\hline \multirow{5}{*}{ Autorreporte } & Agresión & $-0.1(0.91)$ & $0.49(0.98)$ & $0.62(1.36)$ & $0.13(1.15)$ & $9.18 * *$ \\
\hline & Popularidad & $0.13(0.97)$ & $0.46(0.8)$ & $-0.29(1.05)$ & $-0.86(1)$ & $14.46^{* *}$ \\
\hline & "Olympian" & $0.07(0.96)$ & $0.59(0.97)$ & $-0.25(1.34)$ & $-0.49(0.93)$ & $6.85 * *$ \\
\hline & Competencia académica & $0.01(0.96)$ & $0.07(1.13)$ & $-0.34(1.15)$ & $-0.03(1.04)$ & 1.7 \\
\hline & Filiación & $0.05(0.98)$ & $0.03(1.10)$ & $-0.51(1.04)$ & $-0.15(0.9)$ & $4.78^{*}$ \\
\hline
\end{tabular}

$* * p<0.001, * p<0.01$

Fuente: elaboración propia. 


\section{Análisis centrados en la variable}

Considerando que la variable victimización fue construida con base en el consenso de pares sobre el grado de victimización de cada participante, el análisis centrado en la variable la definió como una variable continua. El interés por testear un modelo predictivo de factores de riesgo fundamentó la elección de técnicas analíticas de regresión lineal. Un primer modelo de regresión simple mostró que la victimización es una característica estable $(\beta=0.498, t=13.92, p<0.001)$. Con el objetivo de identificar variables predictoras, se llevaron a cabo tres análisis de regresión jerárquica en los cuales la variable dependiente fue la victimización en el tiempo 2 (segunda medición). Las variables independientes fueron, en el primer paso, la victimización en el tiempo 1 (primera medición) para controlar el efecto de la estabilidad y en el segundo paso, (método stepwise) todas las características en el tiempo 1 según el reporte de pares, profesores y autorreportadas, para cada uno de los modelos.

Según el reporte de pares los predictores de la victimización después de un período de un año -por sobre el efecto de la estabilidad- son la preferencia social $(\beta=-0.15, t=-3.59, p<0.001)$ y las Conductas prosociales $(\beta=-0.11, t=-2.96, p<0.01)$ (ambos efectos negativos; $r_{\text {total }}^{2}=0.29$ ); en otras palabras, estudiantes rechazados socialmente y con bajas conductas prosociales percibidas por sus pares tienen mayor riesgo de ser victimizados. Análisis similares según el reporte de profesores muestran como predictores a las Dificultades psicológicas $(\beta=0.13, t=2.43, p<0.05)$ y las características "Olímpicas" (Olympian, efecto negativo; $\beta=-0.10$, $t=-2.11, p<0.05$ ); estudiantes caracterizados por sus profesores como tristes, ansiosos y sin características olímpicas, sufrirían en mayor grado de victimización $\left(r_{\text {total }}^{2}=0.29\right)$. Finalmente, ninguna característica autorreportada fue significativa como predictora de la victimización $\left(r_{\text {total }}^{2}=0.28\right)$.

¿Existen diferencias de género respecto de los factores de riesgo asociados a la victimización? Para responder a esta pregunta, se repitieron los análisis de regresión jerárquica para mujeres (reportados en Tabla 3) y hombres (Tabla 4) por separado.

Como se puede observar, para las víctimas mujeres los predictores de la victimización luego de un período de un año, y por sobre el efecto de la estabilidad, serían según el reporte de pares la preferencia social (efecto negativo; $\beta=-0.19, t=-3.63, p<0.001$ ) y la competencia académica según el reporte de profesores (efecto negativo; $\beta=-0.18, t=-3.00, p<0.01$ ). En otras palabras, mujeres rechazadas socialmente según sus pares, y con bajas competencias académicas según sus profesores, estarían en mayor riesgo de ser victimizadas por sus pares.

TABLA 3

Modelos de regresión jerárquica sobre la estabilidad y predictores de la victimización según reporte de pares, profesores y autorreporte: Mujeres

\begin{tabular}{llccccc}
\hline & Reporte de pares & B & SE B & $\beta$ & $t$ & $R^{2}$ \\
\hline Paso 1 & (Constante) & -0.09 & 0.05 & & $-2.00^{*}$ & \\
& Victimización tiempo 1 & 0.34 & 0.06 & 0.33 & $5.75^{* * *}$ & 0.18 \\
& & & & & & \\
Paso 2 & Preferencia social & -0.19 & 0.05 & -0.21 & $-3.63^{* * *}$ & 0.21 \\
& & & & & & \\
Variables excluidas & Conducta prosocial & & & -0.1 & -1.83 \\
& Popularidad & & -0.04 & $<1$ \\
& Agresión & & -0.03 & $<1$ \\
& Prominencia social & & & -0.01 & $<1$ \\
\hline
\end{tabular}




\begin{tabular}{|c|c|c|c|c|c|c|}
\hline \multicolumn{2}{|c|}{ Reporte de pares } & $B$ & SE B & $\beta$ & $t$ & $R^{2}$ \\
\hline \multicolumn{7}{|l|}{ Reporte de profesores } \\
\hline \multirow[t]{2}{*}{ Paso 1} & (Constante) & -0.13 & 0.05 & & $-2.44 *$ & \\
\hline & Victimización tiempo 1 & 0.36 & 0.06 & 0.36 & $6.14 * * *$ & 0.17 \\
\hline Paso 2 & Competencia académica & -0.18 & 0.06 & -0.18 & $-3.00 * *$ & 0.20 \\
\hline \multirow[t]{5}{*}{ Variables excluidas } & Dificultades psicológicas & & & 0.08 & 1.33 & \\
\hline & "Olympian" & & & -0.08 & -1.22 & \\
\hline & Agresión & & & -0.02 & $<1$ & \\
\hline & Popularidad & & & 0.02 & $<1$ & \\
\hline & Conductas prosociales & & & -0.01 & $<1$ & \\
\hline \multicolumn{7}{|l|}{ Autorreporte } \\
\hline \multirow[t]{2}{*}{ Paso 1} & (Constante) & -0.1 & 0.05 & & -1.95 & \\
\hline & Victimización tiempo 1 & 0.48 & 0.06 & 0.47 & $8.29 * * *$ & 0.22 \\
\hline \multirow[t]{5}{*}{ Variables excluidas } & Agresión & & & 0.06 & 1.01 & \\
\hline & Competencia académica & & & -0.05 & $<1$ & \\
\hline & "Olympian" & & & -0.04 & $<1$ & \\
\hline & Popularidad & & & -0.01 & $<1$ & \\
\hline & Filiación & & & 0 & $<1$ & \\
\hline
\end{tabular}

$* p<0.05 ; * p<0.01 ; * * p<0.001$

Nota. Los valores de las variables en el paso 1 reflejan los valores obtenidos después de la inclusión de las otras variables que alcanzaron el valor de selección de $p<0.05$.

Fuente: elaboración propia.

Análisis similares para los hombres muestran un resultado distinto. En el caso de ellos, los factores de riesgo para la victimización son, tanto según el reporte de pares como de profesores, la popularidad (efectos negativos; $\beta=-0.23, t=-4.13 ;$ y $\beta=-0.2$, $t=-3.28$, ps $<0.001)$. Es decir, la baja popularidad favorecería el ser victimizado por sus pares, tanto desde la perspectiva de los propios compañeros como la de los profesores.

\section{TABLA 4}

Modelos de regresión jerárquica sobre la estabilidad y predictores de la victimización según reporte de pares, profesores y autorreporte: Hombres

\begin{tabular}{lcccccc}
\hline & Reporte de pares & B & SE B & $\beta$ & $t$ & $R^{2}$ \\
\hline Paso 1 & (Constante) & 0.1 & 0.05 & & 1.88 & \\
& Victimización tiempo 1 & 0.45 & 0.05 & 0.47 & $8.86^{* * *}$ & 0.32 \\
& Popularidad & -0.23 & 0.06 & -0.22 & $-4.13 * * *$ & 0.36 \\
Paso 2 & & & &
\end{tabular}




\begin{tabular}{|c|c|c|c|c|c|c|}
\hline \multicolumn{2}{|c|}{ Reporte de pares } & \multirow[t]{2}{*}{$B$} & \multirow[t]{2}{*}{ SE B } & \multirow{2}{*}{$\frac{\beta}{-0.11}$} & \multirow{2}{*}{$\begin{array}{c}\boldsymbol{t} \\
-1.82\end{array}$} & \multirow[t]{2}{*}{$R^{2}$} \\
\hline Variables excluidas & Preferencia social & & & & & \\
\hline & Conducta prosocial & & & -0.06 & -1.02 & \\
\hline & Agresión & & & 0.03 & $<1$ & \\
\hline & Prominencia social & & & -0.03 & $<1$ & \\
\hline \multicolumn{7}{|l|}{ Reporte de profesores } \\
\hline \multirow[t]{2}{*}{ Paso 1} & (Constante) & 0.11 & 0.05 & & $2.03 *$ & \\
\hline & Victimización tiempo 1 & 0.53 & 0.05 & 0.55 & $11.05 * * *$ & 0.34 \\
\hline Paso 2 & Popularidad & -0.2 & 0.06 & -0.16 & $-3.28 * * *$ & 0.37 \\
\hline \multirow[t]{5}{*}{ Variables excluidas } & Dificultades psicológicas & & & 0.08 & 1.44 & \\
\hline & Conductas prosociales & & & 0.05 & $<1$ & \\
\hline & Competencia académica & & & -0.01 & $<1$ & \\
\hline & Agresión & & & 0.01 & $<1$ & \\
\hline & "Olympian" & & & 0 & $<1$ & \\
\hline \multicolumn{7}{|l|}{ Autorreporte } \\
\hline \multirow[t]{2}{*}{ Paso 1} & (Constante) & 0.07 & 0.05 & & 1.22 & \\
\hline & Victimización tiempo 1 & 0.53 & 0.05 & 0.57 & $10.81 * * *$ & 0.33 \\
\hline \multirow[t]{5}{*}{ Variables excluidas } & Popularidad & & & -0.05 & $<1$ & \\
\hline & Agresión & & & 0.04 & $<1$ & \\
\hline & "Olympian" & & & 0.01 & $<1$ & \\
\hline & Competencia académica & & & -0.01 & $<1$ & \\
\hline & Filiación & & & 0 & $<1$ & \\
\hline
\end{tabular}

$p<0.05 ; * * p<0.01 ; * * *<<0.001$

Nota. Los valores de las variables en el paso 1 reflejan los valores obtenidos después de la inclusión de las otras variables que alcanzaron el valor de selección de $p<0.05$.

Fuente: elaboración propia.

\section{Discusión}

A pesar de la creciente cobertura que ha recibido el tema de la victimización entre pares en el contexto escolar, su abordaje ha sido limitado y sesgado desde perspectivas psicologicistas (intentando identificar características de personalidad asociadas a la victimización) o bien centrado en el sujeto agresor. El presente estudio integra elementos que enriquecen la comprensión de este fenómeno y dan luces para posibles estrategias de prevención. En primer lugar, su carácter longitudinal permite identificar distintas trayectorias que los estudiantes pueden seguir respecto de la victimización, integrando así características tanto psicológicas como sociales. Asimismo, la utilización de dos aproximaciones analíticas complementarias -un análisis centrado en el individuo y un análisis centrado en la variable-, permite comprender simultáneamente tanto los posibles perfiles personales asociados a la victimización como la manera en que las variables se comportan a lo largo del tiempo y se integran en función de este tipo de relaciones interpersonales (Cairns \& Rodkin, 1998). Por último, el uso de in- 
formantes múltiples permite una mayor riqueza en la información recogida y otorga una perspectiva más atingente a la realidad escolar, en la cual la percepción de todos los actores influye en los procesos sociales allí presentes (Gest, 2006; Juvonen, Nishina \& Graham, 2001).

Cuatro preguntas guiaron esta investigación. ¿Quiénes son los y las estudiantes victimizados por sus pares? Respecto de esta primera pregunta, los resultados confirman estudios anteriores que retratan a las víctimas con un perfil socioconductual caracterizado por niveles sobre el promedio en agresividad, bajos niveles de estatus social, menores rendimientos académicos e indicios de dificultades en el área psicosocial. Este perfil integra elementos desde las percepciones de los distintos actores, y si bien no constituye necesariamente la presencia de psicopatología, llama la atención sobre la necesidad de considerar las posibles consecuencias de la experiencia de victimización en el desarrollo de los y las adolescentes. En otras palabras, los y las estudiantes victimizados constituyen un grupo de riesgo, presentando un perfil psicosocial caracterizado por potenciales desajustes en diversas áreas. Lo anterior refuerza la necesidad de focalizar medidas preventivas frente a la victimización -y promocionales de relaciones interpersonales positivas- así como de medidas remediales para aquellos estudiantes en dicha situación.

La segunda pregunta abordó la presencia de distintas trayectorias de victimización (Goldbaum et al., 2003) y potenciales perfiles asociados a estas. Aprovechando el diseño longitudinal del estudio, la inclusión de esta perspectiva refuerza el hecho que existen diferentes experiencias de victimización (Berger \& Rodkin, 2009; Rodkin \& Berger, 2008). Los resultados de esta investigación muestran que el perfil psicosocial negativo asociado a la victimización se observa en aquellos estudiantes que pasan a ser victimizados luego de no serlo, o bien en aquellos estudiantes que son victimizados de manera estable a lo largo del tiempo. En el caso del primer grupo, las dificultades sociales, psicológicas, académicas y conductuales pueden deberse al alto impacto afectivo que implica el constituirse en el blanco de las agresiones de los compañeros
(Bierman, 2004) y a la necesidad de hacer frente a esta situación con el distrés asociado. Respecto de las víctimas estables, se puede pensar también que estos estudiantes no tengan recursos o herramientas adecuadas para poder hacer frente a estas experiencias (Boxer, Goldstein, Musher-Eizenman, Dubow \& Heretick, 2005), sean invisibilizados por la cultura escolar (Rodkin \& Berger, 2008), o se hayan tornado funcionales a la cultura de pares imperante (Berger, 2008; Nishina, 2004; Prinstein \& Cillessen, 2003). Cabe destacar también que las víctimas resilientes (aquellas que dejaron dicha posición) presentan altos niveles de agresividad, lo cual hace cuestionarse sobre el costo social de salir de la victimización (Kochenderfer-Ladd, 2003).

¿Es la victimización una característica estable en el tiempo? Los resultados del presente estudio muestran que la victimización es estable. Esto, sumado a lo antes mencionado respecto del perfil distintivo de las víctimas estables, releva la necesidad de desarrollar instrumentos y metodologías apropiados para la identificación de los y las estudiantes en esta situación. El hecho de que la victimización sea estable favorece la sensación de desesperanza y la atribución interna respecto de esta situación (Graham \& Juvonen, 1998), potenciando así el círculo del silencio y de la victimización (Barudy, 1999). No obstante lo anterior, los resultados muestran que no todos los niños victimizados mantienen dicho estatus (víctimas llamadas resilientes en este estudio). Esto abre dos importantes líneas de desarrollo e investigación futuras. Primero, plantea la necesidad de que profesionales del área de la salud y la educación trabajen con niños y niñas victimizadas, intentado favorecer un afrontamiento positivo de esta situación y su superación definitiva. Por otra parte, enfatiza la necesidad de investigar los factores preventivos, considerando aquellos elementos que favorecen en un estudiante la capacidad de superar esta situación, en el grupo el establecimiento de relaciones interpersonales positivas, o bien en una institución las estrategias y mecanismos para abordar los casos de victimización y generar ambientes protectores (Berger \& Lisboa, 2008).

La última pregunta que guió este estudio precisamente se abocó a la identificación de factores 
de riesgo que, como plantea Rutter (2006), pueden constituir la mirada inversa a los factores protectores y por tanto igual de iluminadora para estrategias de intervención. Ahora bien, la evidencia acumulada respecto de las diferencias de género en la victimización entre pares (Berger \& Rodkin, 2009; Duncan, 1999; Espelage et al., 2004; Stein, 1995) aconsejó el análisis por separado para hombres y mujeres. Los resultados muestran primero que pares y profesores perciben distintas características como factores de riesgo para la victimización, situación de gran relevancia si se considera que son los profesores quienes enfrentan en primera instancia las situaciones de matonaje, y quienes implementan medidas para afrontar estas situaciones. En la medida en que las percepciones sean diversas, las estrategias que puedan llevarse a cabo desde los profesores pueden ser poco significativas o incluso invalidadas desde los estudiantes, potenciando la sensación de invisibilidad y desesperanza. Lo anterior vuelve a enfatizar la importancia de integrar diferentes fuentes de información y reporte (Flores \& Santoyo, 2009; Troop-Gordon \& Ladd, 2005), pero al mismo tiempo ser cuidadosos con la manera en que dicha información es utilizada. Por ejemplo, diversos estudios han mostrado que el autorreporte en general presenta menor confiabilidad en estudios similares, ya que puede basarse más en un estado emocional transitorio que en una característica estable, y no necesariamente constituir una percepción compartida por el entorno social (Graham, Bellmore \& Juvonen, 2003; Huizinga \& Elliott, 1986; Juvonen et al., 2001).

Las diferencias observadas entre hombres y mujeres remiten a estereotipos tradicionales de género. Para las mujeres, el reporte de pares señala a la preferencia social como un factor protector, mientras que para los hombres es la popularidad el factor principal de protección. Si bien ambas características apuntan al estatus social, lo hacen desde perspectivas muy distintas; la primera desde la deseabilidad y cercanía, la segunda, desde la prominencia y el poder. Por otra parte, llama la atención que para los profesores este estereotipo se mantenga para sus alumnos hombres, identificando a la popularidad como factor protector. En otras palabras, niños percibidos por sus profesores como populares no serían victimizados, validando de manera implícita la prominencia y poder social como una estrategia defensiva. En cambio para las mujeres, es la competencia académica la que actúa como protección desde la perspectiva de los profesores, situación que posiblemente remite a la percepción de estas alumnas como potenciales apoyos para sus compañeros y compañeras (esto último requiere mayor investigación para poder ser interpretado de manera adecuada). Particularmente para las niñas victimas de agresión entre pares, la discordancia entre el reporte de profesores y pares refuerza el riesgo de este grupo de ser invisibilizado, posiblemente debido a la imagen tradicional del matonaje como una situación entre hombres y/o de violencia física o visible (Berger \& Rodkin, 2009; Duncan, 1999).

El presente estudio presenta algunas limitaciones que deben ser consideradas al interpretar los resultados. En primer lugar, no existe consenso respecto de la mejor manera de identificar a las víctimas. La consideración de un puntaje de corte de una desviación estándar por sobre el promedio es un procedimiento que puede incluir en una misma categoría distintos grados y perfiles de alumnos victimizados. En este sentido, en este trabajo se utilizó tanto un enfoque analítico centrado en las personas como en las variables, para minimizar estas dificultades. Por otra parte, si bien el estudio adopta un modelo longitudinal, dos mediciones no permiten un análisis acabado de posibles trayectorias ni de relaciones causales. Para hacer frente a lo anterior, en la actualidad se está realizando un estudio de seguimiento a los participantes de esta investigación. Un tercer aspecto dice relación con las distintas fuentes de reporte antes mencionado, que si bien constituye una fortaleza, sus resultados e integración deben ser interpretados con cautela. Por último, un desafío metodológico se presenta respecto de la naturaleza de los datos obtenidos a través de nominaciones de pares, la cual generalmente no responde a una distribución normal dada la cantidad de estudiantes que no reciben nominaciones en determinados ítems. El intento por normalizar dichas variables en este estudio sigue el modelo 
de estudios previos (Cillessen \& Mayeux, 2004; Rodkin \& Berger, 2008); no obstante, se requiere desarrollar dispositivos metodológicos que permitan superar esta dificultad.

Los resultados presentados abren importantes líneas de investigación y fortalecen el trabajo realizado por investigadores en diversos países. En primer lugar, la necesidad de estudios longitudinales que permitan comprender la dinámica de la victimización desde una mirada de desarrollo se hace esencial, para lo cual como se mencionó anteriormente se trabaja en un seguimiento de los participantes de este estudio. Por otra parte, se enfatiza la relevancia de la experiencia subjetiva y diversa respecto de este fenómeno, lo cual levanta la necesidad de estudios cualitativos que permitan acceder a los sentidos y significados que la violencia toma en el marco de la cultura de pares, y de manera transversal a la experiencia adolescente (García, 2008). Por último, se destaca la necesidad de continuar levantando evidencia y marcos comprensivos desde la realidad de países latinoamericanos, con el fin de contrastar los modelos y evidencias surgidas en contextos norteamericanos y europeos e ir acumulando un saber pertinente a nuestras realidades para poder generar intervenciones atingentes a nuestras poblaciones escolares (Berger \& Lisboa, 2008).

En síntesis, el presente estudio aporta información relevante y actualizada para comprender la victimización en contextos escolares, desde una perspectiva de desarrollo y comprendiéndola como una forma de relaciones interpersonales que se da en un contexto específico, superando paradigmas que se centren exclusivamente en el individuo y permitiendo abordar la victimización desde una perspectiva ecológica como parte de la dinámica social (Berger, 2008). La distinción de distintas experiencias de victimización, de trayectorias y perfiles socioconductuales asociados, hace necesaria una mirada crítica a las formas en que se da este fenómeno, a las posibilidades de acción de los estudiantes involucrados y a los costos asociados a la misma. Asimismo, hace necesaria una perspectiva que se centre tanto en la prevención de la victimización, como en la promoción de ambientes nutritivos en los cuales estas dinámicas no tengan cabida. La integración de reportes de pares, profesores y autorreporte, permite identificar factores de riesgo en la población adolescente e ilumina líneas de intervención en la prevención del abuso entre pares. Solo una mejor comprensión de este fenómeno permitirá construir contextos escolares en los cuales las relaciones interpersonales promuevan el bienestar de todos sus participantes.

\section{Referencias}

Barudy, J. (1999). Maltrato Infantil. Prevención y Reparación. Santiago: Editorial Galdoc.

Berger, C. (2008). iEs la agresividad adaptativa? Evidencias de su función social en adolescentes chilenos. En C. Berger \& C. Lisboa (Eds.), Violencia escolar: Estudios y posibilidades de intervención en Latinoamérica (pp. 139-160). Santiago: Editorial Universitaria.

Berger, C. \& Lisboa, C. (2008). Hacia una comprensión ecológica de la agresividad entre pares en el microsistema escolar. En C. Berger \& C. Lisboa (Eds.), Violencia escolar: Estudios y posibilidades de intervención en Latinoamérica (pp.59-83). Santiago: Editorial Universitaria.

Berger, C. \& Rodkin, P. (2009). Male and female victims of male bullies: Social status differences by gender and informant source. Sex Roles, 61, 72-84.

Berger, C., Rodkin, P. \& Karimpour, R. (2008). Bullies and victims at school: Perspectives and strategies for primary prevention. En T. Miller (Ed.), School violence and primary prevention (pp. 287-314). New York: Springer-Verlag.

Bierman, K. (2004). Peer rejection: Developmental processes and intervention strategies. New York: Guilford Press.

Boivin, M., Dodge, K. \& Coie, J. (1995). Individualgroup behavioral similarity and peer status in experimental play groups of boys: The social misfit revisited. Journal of Personality and Social Psychology, 69, 269-279.

Boulton, M. J. (1999). Concurrent and longitudinal relations between children's playground behavior and social preference, victimization, and bullying. Child Development, 70, 944-954. 
Boxer, P., Goldstein, S., Musher-Eizenman, D., Dubow, E. \& Heretick, D. (2005). Developmental issues in school-based aggression prevention from a socialcognitive perspective. The Journal of Primary Prevention, 26(5), 383-400.

Buhs, E., Ladd, G. \& Herald, S. (2006). Peer exclusion and victimization: Processes that mediate the relation between peer group rejection and children's classroom engagement and achievement. Journal of Educational Psychology, 98, 1-13.

Cairns, R. \& Rodkin, P. (1998). Phenomena regained: From configurations to pathways. En R. Cairns, L. Bergman \& J. Kagan (Eds.), Methods and models for studying the individual: Essays in honor of Marian Radke-Yarrow (pp. 245-265). Thousand Oaks, CA: Sage.

Cairns, R., Leung, M., Gest, S. \& Cairns, B. (1995). A brief method for assessing social development: Structure, reliability, stability, and developmental validity of the interpersonal competence scale. Behavioral Research and Therapy, 33, 725-736.

Camodeca, M., Goossens, F., Terwogt, M. \& Schuengel, C. (2002). Bullying and victimization among school-age children: Stability and links to proactive and reactive aggression. Social Development, 11, 332-345.

Chile, Ministerio del Interior. (2008). Segunda encuesta nacional de violencia en el ámbito escolar. Santiago: Autor.

Cillessen, A. H. N. \& Mayeux, L. (2004). From censure to reinforcement: Developmental changes in the association between aggression and social status. Child Development, 75, 147-163.

Duncan, N. (1999). Sexual bullying: Gender conflict and pupil culture in secondary schools. New York: Routledge.

Elias, M. \& Zins, J. (2003). Bullying, other forms of peer harassment, and victimization in the schools: Issues for school psychology research and practice. Journal of Applied School Psychology, 19, 1-6.

Egan, S. K. \& Perry, D. G. (1998). Does low self-regard invite victimization? Developmental Psychology, 34, 299-309.

Espelage, D., Mebane, S. \& Swearer, S. (2004). Gender differences in bullying: Moving beyond mean level differences. En D. Espelage \& S. Swearer (Eds),
Bullying in American schools: A social-ecological perspective on prevention and intervention (pp. 1535). Mahwah, NJ: Erlbaum.

Flores, N. \& Santoyo, C. (2009). Estabilidad y cambio de las relaciones sociales entre niños: análisis de mecanismos funcionales. Revista Mexicana de Análisis de la Conducta, 35, 59-74.

García, B. (2008). Familia, escuela y barrio: un contexto para la comprensión de la violencia escolar. Revista Colombiana de Educación, 55, 108-124.

García, M. \& Madriaza, P. (2005). Sentido y sinsentido de la violencia escolar: análisis cualitativo del discurso de estudiantes chilenos. Psykhe, 14, 165-180.

Gest, S. (2006). Teacher reports of children's friendships and social groups: Agreement with peer reports and implications for studying peer similarity. Social Development, 15, 248-259.

Goldbaum, S., Craig, W., Pepler, D. \& Connolly, J. (2003). Developmental trajectories of victimization: Identifying risk and protective factors. Journal of Applied School Psychology, 19, 139-156.

Graham, S., Bellmore, A. \& Juvonen, J. (2003). Peer victimization in middle school: When self- and peer views diverge. Journal of Applied School Psychology, 19, 117-138.

Graham, S., Bellmore, A. \& Mize, J. (2006). Aggression, victimization, and their co-occurrence in middle school. Journal of Abnormal Child Psychology, 34, 363-378.

Graham, S. \& Juvonen, J. (1998). Self-blame and peer victimization in middle school: An attributional analysis. Developmental Psychology, 34, 587-599.

Hartup, W. (1996). The company they keep: Friendships and their developmental significance. Child Development, 67, 1-13.

Hodges, E., Malone, M. \& Perry, D. (1997). Individual risk and social risk as interacting determinants of victimization in the peer group. Developmental Psychology, 33, 1032-1039.

Hodges, E. \& Perry, D. (1999). Personal and interpersonal antecedents and consequences of victimization by peers. Journal of Personality and Social Psychology, 76, 677-685.

Holt, M. \& Espelage, D. (2003). A cluster analytic investigation of victimization profiles among high school students: Are profiles associated with dif- 
ferential psychological and educational outcomes? Journal of Applied School Psychology, 19, 81-98.

Huizinga, D. \& Elliott, D. (1986). Reassessing the reliability and validity of self-report delinquency measures. Journal of Quantitative Criminology, 2, 293-327.

Jiang, X. L. \& Cillessen, A. H. N. (2005). Stability of continuous measures of sociometric status: A meta-analysis. Developmental Review, 25, 1-25.

Juvonen, J., Nishina, A. \& Graham, S. (2001). Self-views $\mathrm{v} / \mathrm{s}$ peer perceptions of victim status among early adolescents. En J. Juvonen \& S. Graham (Eds.), Peer harassment in school: The plight of the vulnerable and victimized (pp. 105-124). New York: Guilford.

Kochenderfer-Ladd, B. (2003). Identification of aggressive and asocial victims and the stability of their peer victimization. Merrill-Palmer Quarterly, 49, 401-425.

Kumpulainen, K., Räsänen, E., Entonen, I., Almqvist, F., Kresanov, K., Linna, S., ...Tamminen, T. (1998). Bullying and psychiatric symptoms among elementary school-age children. Child Abuse and Neglect, 22, 705-717.

LaFontana, K. \& Cillessen, A. (2002). Children's perceptions of popular and unpopular peers: A multimethod assessment. Developmental Psychology, 38, 635-647.

Lisboa, C. \& Koller, S. (2008). Factores protectores y de riesgo para la agresividad y victimización en escolares brasileños: el rol de los amigos. En C. Berger \& C. Lisboa (Eds.), Violencia escolar: Estudios y posibilidades de intervención en Latinoamérica (pp. 161-182). Santiago: Editorial Universitaria.

Miller, T. \& Krauss, R. (2008). School-related violence: Definitions, scope, and prevention goals. En T. Miller (Ed.), School violence and primary prevention (pp. 15-24). New York: Springer-Verlag.

Nansel, T., Haynie, D. \& Simons-Morton, B. (2003). The association of bullying and victimization with middle school adjustment. Journal of Applied School Psychology, 19, 45-62.

Nishina, A. (2004). A theoretical review of bullying: Can it be eliminated? En C. Sanders \& G. Phye (Eds.), Bullying: Implications for the classroom (pp. 36-62). San Diego: Elsevier Academic Press.
Nylund, K., Bellmore, A., Nishina, A. \& Graham, S. (2007). Subtypes, severity, and structural stability of peer victimization: What does latent class analysis say? Child Development, 78, 1706-1722.

Olweus, D. (1993). Bullying at school. Oxford: Blackwell Publishers.

Orpinas, P. \& Horne, A. (2006). Bullying prevention: Creating a positive school climate and developing social competence. Washington, DC: American Psychological Association.

Ortega, R. \& Mora-Merchán, J. (1997). Agresividad y violencia. El problema de la victimización entre escolares. Revista de Educación, 313, 7-27.

Owens, L., Daly, A. \& Slee, P. (2005). Sex and age differences in victimization and conflict resolution among adolescents in a south Australian school. Aggressive Behavior, 31, 1-12.

Paul, J. \& Cillessen, T. (2003). Dynamics of peer victimization in early adolescence: results from a fouryear longitudinal study. Journal of Applied School Psychology, 19, 25-44.

Pellegrini, A. D. (1998). Bullies and victims in school: A review and call for papers. Journal of Applied Developmental Psychology, 19, 165-176.

Pellegrini, A. D., Bartini, M. \& Brooks, F. (1999). School bullies, victims, and aggressive victims: Factors relating to group affiliation and victimization in early adolescence. Journal of Educational Psychology, 91, 216-224.

Prinstein, M. \& Cillessen, A. (2003). Forms and functions of adolescent peer aggression associated with high levels of peer status. Merrill-Palmer Quarterly, 49, 310-342.

Rodkin, P. C. \& Berger, C. (2008). Who bullies whom? Social status asymmetries by victim gender. International Journal of Behavioral Development, 32, 488-501.

Rodkin, P. \& Hodges, E. (2003). Bullies and victims in the peer ecology: Four questions for psychologists and school professionals. School Psychology Review, 32, 384-400.

Rutter, M. (2006). Genes and behavior: Nature-nurture interplay explained. Oxford: Blackwell Publishing.

Scheithauer, H., Hayer, T., Petermann, F. \& Jugert, G. (2006). Physical, verbal, and relational forms of bullying among German students: Age trends, 
gender differences, and correlates. Aggressive Behavior, 32, 261-275.

Schwartz, D., Proctor, L. J. \& Chien, D. H. (2001). The aggressive victim of bullying: Emotional and behavioral dysregulation as a pathway to victimization by peers. En J. Juvonen \& S. Graham (Eds.), Peer harassment in school: The plight of the vulnerable and victimized (pp. 145-174). New York: Guilford.

Solberg, M. E. \& Olweus, D. (2003). Prevalence estimation of school bullying with the Olweus bully/ victim questionnaire. Aggressive Behavior, 29, 239 . 268.

Stein, N. (1995). Sexual harassment in schools: The public performance of gendered violence. Harvard Educational Review, 65, 145-162.

Sullivan, K., Clearly, M. \& Sullivan, G. (2005). Bullying en la enseñanza secundaria. Barcelona: Ediciones CEAC.

Swearer, S. \& Tam, P. (2003). Perceptions and attitudes toward bullying in middle school youth: A developmental examination across the bully/victim continuum. Journal of Applied School Psychology, 19, 63-79.

Tam, F. (2008). The effects of peer victimization on learning: Evidence from Hong Kong. Journal of School Violence, 7, 43-63.

Tapper, K. \& Boulton, M. (2005). Victim and peer group responses to Different forms of aggression among primary school children. Aggressive Behavior, 31, 238-253.

Troop-Gordon, W. \& Ladd, G. (2005). Trajectories of peer victimization and perceptions of the self and schoolmates: Precursors to internalizing and externalizing problems. Child Development, 76, 1072-1091.

Unnever, J. \& Cornell, D. (2004). Middle school victims of bullying: Who reports being bullied? Aggressive Behavior, 30, 373-388.

Veenstra, R., Lindenberg, S., Zijlstra, B., De Winter, A., Verhulst, F. \& Ormel, J. (2007). The dyadic nature of bullying and victimization: Testing a dual-perspective theory. Child Development, 78, 1843-1854. 